\title{
A pocket computer program for collecting forage selection frequency data in the field
}

\author{
RICHARD P. CINCOTTA
}

\section{Abatract}

An algorithm was developed to conduct bite-count sampling employing a programmable pocket computer. The BASIC program was successfully employed to collect forage selection data on rangeland livestock at a remote field site in Tibet. The program features techniques that are applicable to developing programs for sustained frequency data collection using similar battery-powered computers. Pocket computers have been demonstrated to be powerful field tools, and their potential promises to increase as new units become available with larger memories and added features.

Key Words: pocket computer, bite-count technique, livestock forage selection

In 1987 , I was asked by a team of anthropologists to determine the botanical composition and quality of summer diets of yak, goats, and sheep in a high altitude Tibetan pastoral ecosystem. The bite-count technique (Wallmo and Neff 1970, Hobbs et al. 1979) was chosen for these purposes over the use of an esophageal cannula, thus avoiding surgery on indigenous stock that might have been culturally unacceptable to local pastoralists. I also decided to minimize the amount of labor involved in the bite-count technique by using a relatively inexpensive, battery-powered, programmable pocket computer, which could be easily used on foot or on horse at the remote field site. The objective of this article is to present the algorithm ${ }^{2}$, and the programming techniques that permitted successful data collection. Secondly, I will touch on the present state of the art in pocket computing and its future for field researchers.

\section{Materials and Methods}

\section{Computer}

The pocket computer that I used for frequency data collection was the Sharp ${ }^{1}$ EL-5400, which measures $170 \mathrm{~mm} \times 72 \mathrm{~mm} \times 9.5$ $\mathrm{mm}$, weighs about $125 \mathrm{~g}$, and features a single row, 16-character liquid crystal display. This computer is driven by a 4 bit microprocessor, contains 17.4 kbytes of system ROM (including BASIC interpreter), and 1962 bytes of RAM (including 208 bytes of fixed memory area and 1462 bytes of "user RAM"). RAM is nonvolatile, i.e., when the processor is powered off, memory-resident programs and data are preserved.

Sharp, Tandy, and Hewlett-Packard market a range of flat, battery-powered computers that can be described as pocket computers, but vary widely in size of RAM, keyboard functions, display screen, and price (from about $\$ 60$ to $\$ 130$ ). Present limitations in the size of RAM (even new models with 8 kbytes RAM) make these computers suitable primarily for data collection where only

\footnotetext{
Author is post-doctoral scholar, Department of Anthropology, University of California, Los Angeles $90024-1553$.

The field work during which the program was developed and tested was funded in part by the National Geographic Society, the National Academy of Sciences Committee on Scholarly Communication with the People's Republic of China, and the Tibetan Academy of Social Sciences.

Manuscript accepted 23 January 1989.

This article is not a product review. The inclusion of trade names does not imply an endorsement by the Journal of Range Management.

${ }^{2}$ The algorithm has undergone some minor changes subsequent to field use at the suggestion of two anonymous reviewers.
}

summary data are stored. Applications that require the collection of many individually stored data are impractical with pocket computers similar to the EL-5400. In most models, data can be uploaded for storage on an audio cassette recorder or output to a special attachable printer. However, presently there is no commercially available communications interface for uploading to microcomputers.

\section{Data Collection}

A researcher using the bite-count technique records the particular plant species, plant part (leaf, stem, inflorescence, seeds, whole plant), and condition (green, dry) that an herbivore selects during a predetermined foraging bout (Hobbs et al. 1979). If forage samples are needed for laboratory analysis, vegetation can be plucked either simultaneously (Hobbs et al. 1979) or directly following a "foraging bout" (Coppock et al. 1986), though the simultaneous hand plucking of samples is more likely to produce a sample similar to dietary forage (Van Soest 1982).

During the Tibetan research, summer forage selection was assessed over 1 month from a regularly rotating schedule that comprised fifteen 30 -minute foraging bouts per livestock species. One livestock species was sampled during each bout by recording the selections of 3 individuals, one at a time, during consecutive 10 minute periods. Between 400 and 700 bites of forage were recorded during each bout. At the end of each session, the observer recorded the date, time, livestock species, location, vegetation type, relative frequency of each item, and total bite count in a field notebook. Following a bout, $100 \mathrm{~g}$ live weight duplicates of forage, in the proportions selected during the bout, were hand plucked and bagged at the site of grazing. Bite weight samples were collected and bite rates were estimated to facilitate the computation of dry-matter basis botanical composition of the diet (Baker and Hobbs 1982).

Throughout this research it was necessary to count bites at distances between 5 and $10 \mathrm{~m}$. using $8 x$ binoculars, especially with skittish sheep and goats that were foraging on small alpine-desert plants. This situation may commonly arise when working among pastoralist societies where herders propel stones to herd small stock. If data were collected unassisted under these conditions, an audio recorder and microphone would have been necessary, since the attention and hands of the observer were occupied using the binoculars. In this field situation, however, the observer was assisted by a field technician who input data on the pocket computer as the observer called out item numbers.

\section{Program}

A program for collecting bite-count data, e.g. BITECOUNT (Fig. 1), must store and display on command (1) the sum of bites, (2) the count of bites (absolute frequency) for each item selected, (3) and the proportion (relative frequency) attributed to each item. To use the program, BITECOUNT, names of dietary items must be coded, as positive integers $(1,2, \ldots, M$; where the constant, $M$, is the maximum; $M$ can be changed by editing the program), prior to sampling. A list of codes may be written on a file card that is weather-proofed with transparent tape and clipped to the observer's 


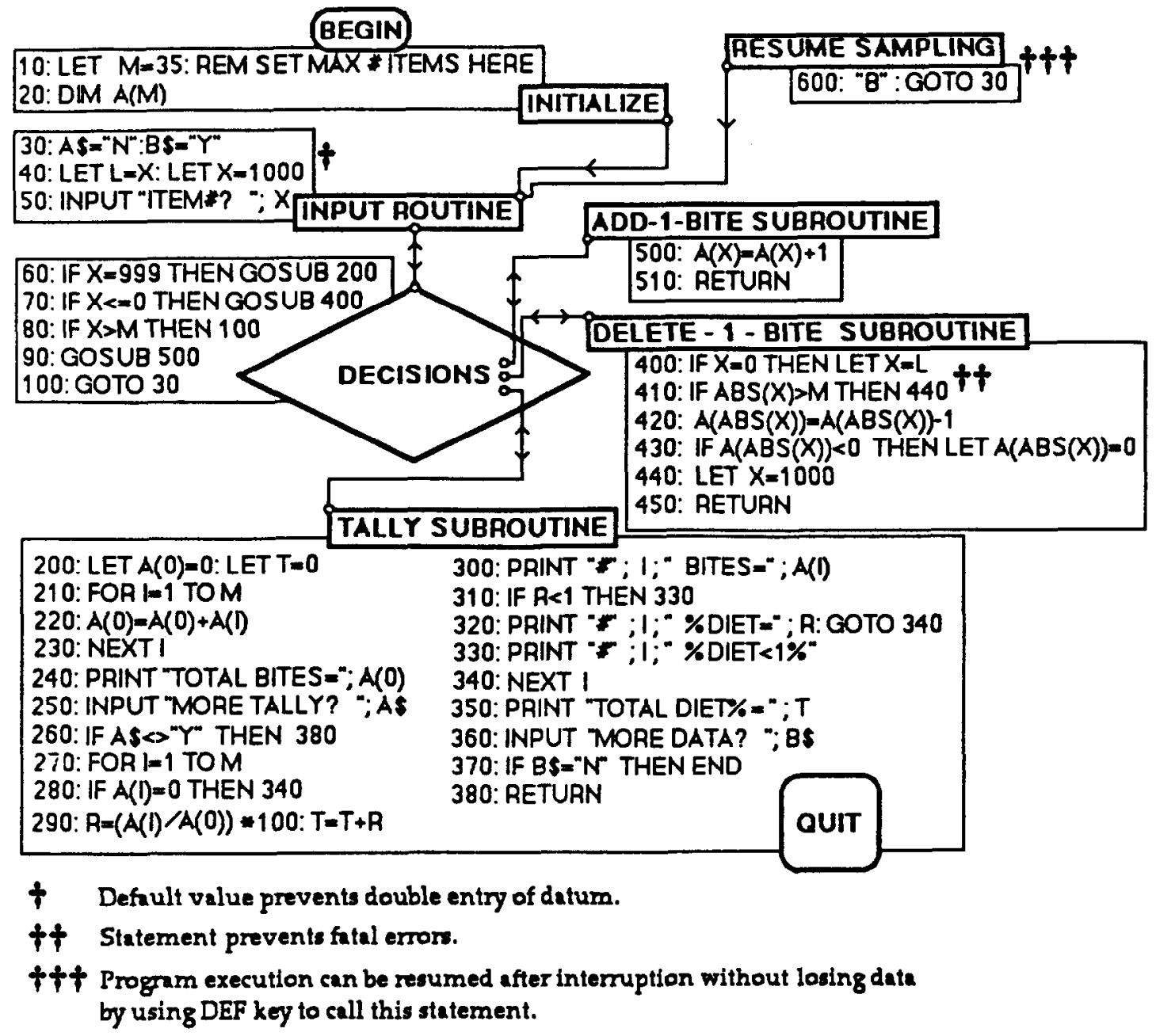

Fig. 1. BASIC code of a pocket computer program for collecting livestock forage selection data using the bite-count technique. This program runs on the Sharp EL-5400, but with minor changes will run on similar pocket computers.

pocket. Observed bites are entered at a prompt, ITEM\#?, which calls for the input of the code number of the dietary item selected. At each input bite, BITECOUNT adds 1 to the item's counter, which is saved as an element of a one-dimensional array variable. A bite mistakenly input can be deleted on the following entry by entering 0 , or at any other input prompt by entering the negative of the incorrectly entered item number.

At any time, the user can invoke a summary (tally) by entering 999 at the input prompt. First, BITECOUNT displays the total number of bites presently recorded, and then prompts with $M O R E$ $T A L L Y$ ?, giving the user the option to resume sampling. If the tally is continued, the program displays only those diet items that were input during the sample, each with its respective absolute and relative frequencies. As an error check, BITECOUNT displays the sum of relative frequencies (which should be approximately $100 \%$ ). At the next prompt, MORE DATA?, the user may resume input or terminate the program.

\section{Results}

Although Tibetan field technicians with whom I worked had never before used a programmable computer, they found BITECOUNT easy to run after a brief demonstration. The two small lithium type cells required to power the pocket computer lasted for the entire 3 months (and well beyond) of continual programming, editing, and data entry with BITECOUNT, as well as unrelated statistical and arithmetic calculations. Exposure to light rainfall did not affect the performance of the pocket computer.

Observers using the bite-count technique have generally chosen to record samples on a battery powered audio cassette recorder, or by pencil and paper. BITECOUNT eliminated lengthy transcription of audio casettes in the field, and reduced the required amount of data recording on paper.

\section{Discussion}

\section{Programming Considerations}

The success of BITECOUNT was due to several programming techniques (Fig. 1) that (1) filtered erroneous input; (2) avoided fatal errors; and (3) permitted resumption of program execution without loss of data following unintentional program termination or "power off" status.

Certain intuitive approaches to data collection that succeed in the 64 kbytes of RAM allocated to interpreted BASIC program storage in microcomputers (PC's and compatibles) are unacceptable in pocket computer BASIC. For example, recording plant part and condition for a list of species would be most efficiently stored in a three-dimensional array. However, in pocket computer BASIC only one- and two- dimensional arrays are permissible. Since each array storage location requires a minimum of 15 bytes of RAM (for a numeric variable), it is easy to imagine how quickly the limited available memory can be consumed by a two- 
dimensional array. Thus, it is advisable to limit array variables to one dimension unless absolutely necessary. Memory constraints will undoubtedly be less of a problem with larger RAM's and expansion kits offered in newer models.

\section{The Future for Pocket Computers}

Pocket computers have served as useful tools for physicians in both laboratory (Ledochowski et al. 1985) and clinical settings (Lorentz et al. 1987, Childs and Pang 1988, Castellano et al. 1986). For sequential data collection and field analyses requiring storage of lengthy programs and intermediate files, a different class of machine referred to as "hand-held computers" have proved useful, especially in agricultural field experiments (Law and Reeves 1984, Kidger and McNicol 1986, Jackson and Stone 1987). Hand-held computers are generally equipped with at least $16 \mathrm{kbyte}$ RAM, full keyboard, real-time clock, and have the capability to communicate with microcomputers (various hand-held models appropriate for field data collection are reviewed by Elias 1984). These computers have the disadvantages of a larger size (usually brief case size) and weight (around $2 \mathrm{~kg}$ ), and higher cost (from $\$ 400$ to above $\$ 2,500$ ).

As one might have expected from observing the dynamism of the computer industry, the advantages of both pocket and hand-held computers have been merged into a new breed of fully transportable and programmable computer. For example, the Psion Organiser II Model XP, which is marketed as a pocket "personal organizer and diary", measures $142 \mathrm{~mm} \times 78 \mathrm{~mm} \times 29 \mathrm{~mm}$ and weighs $225 \mathrm{~g}$. This machine features a 16 kbyte RAM (expandable to 128 kbyte), real-time clock, and optional RS232 cable that connects the Organiser II to either a serial port of a MS-DOS microcomputer for uploading and downloading programs and data, or directly to a serial printer. Software is available for PC's and compatibles that emulates the Organiser II's BASIC-like language interpreter, allowing the user to develop and test software in the familiar MS-DOS environment before downloading to the smaller computer.

As pocket computer technology improves, field researchers will have at their disposal the information handling capabilities that are presently thought the domain of much larger machines. It will require a balance of imaginative programming and scientific communication to fully realize the potential that computer-aided field data collection holds for natural resources management and research.

\section{Literature Cited}

Baker, D.L., and N.T. Hobbs. 1982. Composition and quality of elk summer diets in Colorado. J. Wildl. Manage. 46:694-703.

Castellano, J., J.E. Toher, M.J. Sindler, and J.D. Dumler. 1986. Pocket computer for real-time ultrasound. South Med. J. 79:1331-1336.

Childs, G.E., and L. Pang. 1988. Analysis of dose response curves for the in vitro susceptibility of Plasmodium falciparum to antimalarias using a pocket computer. Amer. J. Trop. Med. Hyg. 38:15-18.

Coppock, D.L., J.E. Ellis, and D.M. Swift. 1986. Livestock feeding ecology and resource utilization in a nomadic pastoral ecosystem. J. Appl. Ecol. 23:585-595.

Elias, M.F. 1984. Handheld computers for recording timed behavior observations. Ethol. Sociobiol. 5:59-60.

Hobbs, N.T., D.L. Baker, J.E. Ellis, and D.M. Swift. 1979. Composition and quality of elk diets during winter and summer: a preliminary analysis. p. 47-53. In: M.S. Boyce and L.D. Hayden-Wing (eds.). North American elk: ecology, behavior and management. University of Wyoming, Laramie.

Jackson, H.O., and J.A. Stone. 1987. Improved data collection program for a notebook-sized microcomputer. Agron. J. 79:1087-1089.

Kidger, R., and J.W. McNicol. 1986. A program for data recording on a portable microcomputer. Computers and Electronics in Agriculture 1:213-217.

Law, J.R., and J.C. Reeves. 1984. The use of hand-held data terminals in National Institute of Agricultural Botany [UK] field trial recording. J. Nat. Inst. Agr. Bot. 16:397-414.

Ledochowski, M., M. Herold, and F. Dienstl. 1985. A pocket computer program for differential diagnosis of tumor-induced hypercalcemia and primary hyperarathyruidism. Comput. Method. Program. Biomed. 21:55-56.

Lorentz, A., P. Gasteiger, and P.M. Osswald. 1987. Calculation of the allowable pretransfusion blood loss with a programmable pocket computer. Anaesthesist 36:306-312.

Wallmo, O.C., and P.J. Neff. 1970. Direct observation of tamed deer to measure their consumption of natural forage. p. 105-110. In: Range and Wildlife Habitat Evaluation: A Research Symposium, USDA Forest Serv. Misc. Pub. 1147.

Van Soest, P.J. 1982. Nutritional ecology of the ruminant. O\&B Books, Corvallis, Ore. 